\title{
Una aproximación teórica a la ilusión del control y la importancia de la (des)confianza en las alianzas estratégicas
}

A theoretical approach to the illusion of control and the importance of (dis)trust in strategic alliances

\author{
Germán Velásquez Salazar \\ Pontificia Universidad Católica del Perú \\ gvelasquezs@pucp.pe
}

\begin{abstract}
Esta investigación pretende explicar los efectos de la participación de la confianza y la desconfianza, como características independientes de los mecanismos de control decididos por las políticas de gobernanza de las firmas y generados por el deseo de control de las firmas. Nuestra revisión de la literatura ha identificado que no hay claridad sobre la influencia de la confianza o desconfianza entre las firmas como mecanismos de control orientados al logro de los objetivos de una alianza estratégica.
\end{abstract}

Palabras clave: control, alianzas estratégicas, confianza, desconfianza

This research aims to explain the effects of the participation of trust and distrust, as independent characteristics of the control mechanisms decided by the governance policies of the firms and generated by the desire for control of the firms. Our review of the literature has identified that there is no clarity on the influence of trust or distrust between firms as control mechanisms aimed at achieving the objectives of a strategic alliance.

Keywords: control, strategic alliances, trust, distrust 


\section{Introducción}

Este artículo analizará la complejidad de las alianzas estratégicas y cómo la confianza y la desconfianza moderan los mecanismos de control de sus miembros para el desempeño de la alianza estratégica. Para ello, se presenta una investigación bibliográfica orientada a la influencia del control en la gestión de las alianzas estratégicas. Nuestra revisión de la literatura buscó los aportes de los referentes más citados en la bibliografía de mayor impacto relacionada con las palabras claves. Para Stoner et al. (1996), "control» es el proceso que permite garantizar que las actividades reales se ajusten a las proyectadas. Ballou (2004) complementa la definición incorporándole una acción correctora para acercar la actividad a lo planeado. Partamos de que el control interorganizacional está en discusión en la literatura, siendo los problemas de apropiación y los requisitos de coordinación con el socio fuentes de análisis y explicación para los mecanismos formales e informales diseñados e implementados por las organizaciones para tratar de alcanzar sus objetivos. Este control interorganizacional consta de cuatro elementos, que se derivan de medidas comúnmente utilizadas en los mecanismos de control que incluyen: 1) el establecimiento de objetivos, 2) revisiones generales, 3) intercambio de información y 4) resolución conjunta de problemas. (Dekker, 2004; Christoffersen, 2013; Dekker et al., 2013).

El control se hace relevante en las alianzas estratégicas, conceptualizadas como acuerdos donde dos o más socios independientes entre sí comparten el compromiso de alcanzar sus objetivos al poner en común sus recursos y coordinar sus actividades compartiendo riesgos y costos, lo que demanda el compromiso de los socios para que las empresas cumplan con los acuerdos y compartan los resultados potenciales (Teece, 1992; Li et al., 2013; Jiang et al., 2013). Las organizaciones necesitan de otras organizaciones para el logro de sus objetivos y por eso aparece la necesidad de control en los diferentes roles que desempeñan en las alianzas estratégicas, buscando complementariedad para el logro de sus objetivos particulares y el éxito de la alianza creada, expresando objetiva y subjetivamente las condiciones sobre las cuales se desarrollará la relación entre ellas, y estableciendo sistemas orientados a verificar el cumplimiento de las condiciones del acuerdo (Albers et al., 2016). La presión de las organizaciones que pretenden garantizar que las actividades de la firma coincidan con sus objetivos colisiona con comportamientos no deseados de los sujetos controlados y ello incentiva el deseo de control en las firmas, estimulado por la probabilidad del comportamiento oportunista del socio, lo que -a su vez-provoca políticas de gobernanza definidas principalmente por confianza, compromiso, reputación, experiencia pasada, tamaño del socio, dependencia del socio de sí mismo, poder sobre el socio, ajuste estratégico, consenso y sensación de equidad (Hameed \& Abbott, 2017). Cabe añadir que los académicos no han prestado especial atención al deseo de control, que juega un papel clave en la determinación de cómo las empresas monitorean el negocio de la alianza y revela la existencia de compañías en las que el patrón de decisiones es impulsado por la importancia de tener el control en sus intercambios internos (Tse et al., 2019). Estos estándares de control pueden afectar la eficiencia de la alianza estratégica, son provocados principalmente por la confianza y la desconfianza entre las firmas, y obran como obstáculos para el logro de sus propósitos (Mayer et al., 1995). 
En esta investigación definiremos "confianza» como la voluntad de una parte de ser vulnerable a las acciones de otra, con base en la expectativa de que esta realizará una acción particular importante para la primera, independientemente de su capacidad de monitorear o controlar a la otra parte (Mayer et al., 1995). En este contexto, los antecedentes y las consecuencias de la confianza en las alianzas estratégicas han sido objeto de interés por parte de los estudiosos de las alianzas, y existe consenso en que la confianza juega un papel importante en la explicación del comportamiento de la alianza y, en última instancia, de su éxito (Gulati \& Nickerson, 2008; Zaheer \& McEvely, 1998). Asimismo, debido a que la literatura muestra ambigüedad respecto a la influencia del control en el logro de los objetivos de las firmas, en sus relaciones interorganizacionales y el comportamiento de los socios (Taleb, 2013), nuestra investigación busca explicar la participación de la confianza y la desconfianza como características independientes a los mecanismos de control decididos por las políticas de gobernanza de las firmas, atendiendo también a cómo influencian el éxito de las alianzas estratégicas. Para este propósito, se exploraron diferentes teorías y enfoques metodológicos que muestran aquellas corrientes de investigación sobre alianzas estratégicas que proporcionan una comprensión del fenómeno desde diferentes perspectivas, e identificado las fallas que conducen al fracaso de las alianzas estratégicas, explicando las razones por las que estas no alcanzan los propósitos para los que fueron constituidas (Gomes et al., 2011; Christoffersen, 2013).

\section{Revisión de la literatura y marco teórico}

\subsection{Revisión de la literatura}

En esta sección se ofrece una revisión de literatura, empleando los estudios desarrollados a la fecha que exploran el control en perspectiva interorganizacional. En primer lugar, se presentan las investigaciones especializadas en alianzas estratégicas, para seguir con las aplicaciones del control y sus diferentes enfoques, finalizando con una revisión actualizada de artículos vinculados a la confianza y la desconfianza como instrumentos independientes del control.

\subsubsection{Las alianzas estratégicas en la literatura}

El término "alianza estratégica» se introdujo en la década de 1980 para describir la multitud de formas de acuerdos entre empresas y otras organizaciones, y se refiere esencialmente a acuerdos mediante los cuales dos o más socios comparten el compromiso de alcanzar un objetivo común al contribuir ambos con sus recursos y coordinar sus actividades. Las alianzas estratégicas se definen como acuerdos de cooperación de duración relativa entre organizaciones que involucran flujos de productos, dinero e información para el logro conjunto de metas individuales vinculadas a la misión corporativa de cada organización (Teece, 1992; Lorange \& Roos, 1992; Varadarajan \& Cunningham, 1995; Parkhe, 1998). Estas se establecen para crear valor económico para las empresas asociadas y se considera que la confianza es un impulsor importante del desempeño de la alianza. Por esta razón los administradores de la alianza son importantes, ya que la confianza requiere participación, 
compromiso y el apoyo de los actores involucrados en la alianza estratégica (Zucker, 1986; Parkhe, 1998; Dyer \& Singh, 1998; Anand \& Khanna, 2000; Zollo et al., 2002; Khanna, 1998; Harris, 2006; Lunnan \& Haugland, 2008). Por otro lado, aunque este conocimiento está bien establecido y el número de alianzas recién formadas en todo el mundo aumenta constantemente, observamos altas tasas de fracaso de hasta el 60 \% (Bleeke \& Ernst, 1991; Duysters et al., 1999; Park \& Ungson, 2001; Kapmeier \& Struben, 2017). Por lo tanto, la pregunta a la que se enfrentan los gerentes de alianzas - y que la investigación empírica debería ayudar a responder - es cómo establecer intencionalmente la confianza y fomentar proactivamente su desarrollo en alianzas estratégicas para alcanzar sus objetivos.

Se ha demostrado que la selección de socios es una decisión conscientemente estratégica y específica en la creación de una alianza estratégica, y que la importancia y la variación de los criterios utilizados por una empresa en la selección de un socio reflejan una amplia gama de factores, muchos de los cuales derivan de las necesidades de la empresa (Dacin et al., 1997; Hitt et al., 2000). Por ello, la selección de un socio parte de una empresa para la formación de una alianza estratégica apropiada es una decisión crítica y determina la combinación de habilidades, conocimientos y recursos de una alianza estratégica; sus políticas y procedimientos operativos; y su vulnerabilidad a las condiciones, estructuras y cambios institucionales originales (Hitt et al., 1997; Child \& Faulkner, 1998). Así, la variable clave del desempeño en la relación entre firmas sería la interacción repetida en el tiempo que se presenta entre los socios, implicando los niveles de confianza necesarios para el logro de los objetivos de la alianza (Ceptureanu et al., 2018).

\subsubsection{El rol y los efectos del control}

El control es definido como un sólido predictor del comportamiento, la emoción, la motivación, el desempeño, el éxito y el fracaso de las personas en muchos dominios de la vida. Predice comportamientos y establece relaciones con el rendimiento, la motivación, la competencia interpersonal, el éxito laboral, la creatividad, la resolución de problemas, y la búsqueda y el procesamiento de información (Brim, 1974; Baltes \& Baltes, 1986; Schulz \& Heckhausen, 1991; Ruiz, 1993; Heckhausen \& Schulz, 1995; De Grande, 2014). El control se considera más fácilmente como instrumento de medición; sin embargo, el control percibido no es una construcción aislada o una sola medida.

En la literatura vinculada a la gestión se presentan dos enfoques principales de control. Primero, un enfoque que se centra en el establecimiento y la utilización de reglas, procedimientos y políticas formales para monitorear y recompensar el desempeño deseable; es decir, el control formal. Segundo, un enfoque centrado en el control informal, también llamado social o relacional, que enfatiza el poder regulador de las normas organizacionales, los valores, la cultura y la internalización de metas para promover resultados deseables (Cardinal et al., 2004). Al contrario de los mecanismos formales, los controles informales no se eligen, diseñan o implementan directamente; más bien, se construyen paso a paso durante intercambios repetidos. La confianza y las normas informales (por ejemplo, flexibilidad, solidaridad e intercambio de información) son dos mecanismos principales de control discutidos en la literatura (Liu et al., 2009). Los controles tienen como objetivo mejorar la probabilidad de lograr las metas a través de la colaboración y, por lo tanto, fomentar el 
desempeño. Los controles relacionales se refieren a la medida en que los intercambios se rigen por las relaciones sociales, las estructuras informales y el autoaprendizaje; mientras que los controles organizacionales incluyen todos los mecanismos formales diseñados e implementados por una organización para lidiar con las preocupaciones de apropiación y requisitos de coordinación.

Por otro lado, la relación entre confianza y control puede ser de naturaleza complementaria o sustitutiva, según el tipo de control. El control formal puede socavar la confianza porque el uso de reglas y objetivos estrictos significa que los miembros no tienen la autonomía para decidir qué funciona mejor. En este sentido, la confianza y el control actúan como sustitutos. Esto es consistente con la opinión de que la confianza, en particular la confianza de buena voluntad, reduce la necesidad de diseñar y monitorear las salvaguardas contractuales. El control social, por otro lado, puede complementar la confianza en la medida en que enfatiza la creación de metas y normas compartidas que, probablemente, aumentarán el entendimiento mutuo y generarán confianza (Das \& Teng, 2001).

Asimismo, los mecanismos contractuales reducen el oportunismo y favorecen el desempeño de las relaciones; mientras que los sistemas de medición del desempeño operativo mejoran la calidad del producto, la entrega a tiempo y el ahorro de costos. Los mecanismos organizativos como el establecimiento de objetivos, las revisiones operativas y la retroalimentación están asociados con la ventaja colaborativa, mejores relaciones de calidad y el desempeño general, lo que nos llevaría a asumir que los controles interorganizacionales facilitan el desempeño (Mahama, 2006; Liu et al., 2009; Cao \& Zhang, 2011; Dahlquist, 2015).

A pesar de que las estrategias de control han recibido una atención permanente en la investigación de las alianzas estratégicas, se mantiene una falta de comprensión sobre sus efectos en el desempeño de las alianzas. Las teorías que justifican el uso del monitoreo como mecanismo de control se basan en la lógica de que el monitoreo aumenta las presiones que los involucrados se comporten de acuerdo con las reglas y/o normas. En este contexto, el seguimiento debería tener un impacto positivo en el desempeño. No obstante, se considera que los mecanismos de control son importantes, pero también peligrosos para gestionar el desempeño de las relaciones entre empresas, advirtiendo que las decisiones de monitoreo de un socio pueden afectar sus resultados (Grewal et al., 2010; Zhang \& Zhou, 2013). Una perspectiva controversial nos dice que las previsiones no son neutrales, sino cuestión de iatrogenia en tanto pueden perjudicar totalmente a quienes asumen riesgos. Hay datos empíricos que señalan que ofrecer a alguien una previsión numérica al azar hace que esa persona se arriesgue más, aunque sepa que la previsión es aleatoria (Taleb, 2013).

En resumen, las características cada vez más complejas de las alianzas estratégicas motivan la incorporación de mecanismos de control entre los actores participantes, lo que nos lleva a considerar la siguiente hipótesis:

H1: a mayor complejidad de las alianzas estratégicas, mayor será el nivel de control entre los socios. 


\subsubsection{El efecto de la confianza en las alianzas estratégicas}

La confianza se define como la voluntad de una parte de ser vulnerable a las acciones de otra, con base en la expectativa de que esta realizará una acción particular importante para la primera. La palabra «confianza» es ambigua porque denota tanto la evaluación de ambas partes como la toma de la decisión de confiar en el otro; es decir, la decisión de confiar en el socio constituye un estado mental de confianza unido a la acción de confiar (Mayer et al., 1995).

Para ejercer el acto de confiar es necesario tener competencia y disposición. La confianza se ha estudiado desde perspectivas disciplinarias diferentes. En general, se presenta como un concepto específico en un contexto que es abstracto, elusivo, frágil y efímero. A su vez, desde la perspectiva de los costos de transacción, todo arreglo de colaboración enfrenta la amenaza potencial del oportunismo, lo que hace que las empresas participantes sean vulnerables a las acciones de sus socios. La confianza también puede considerarse como un medio para asegurar la cooperación e inhibir el comportamiento oportunista (Bhattacharya et al., 1998; Zaheer et al., 1998; Young-Ybarra \& Wiersema, 1999; Ybarra \& Turk, 2009; Kramer \& Lewicki, 2010). Así, un alto nivel de confianza reduce los conflictos, provoca una mayor satisfacción del socio y mejora el comportamiento cooperativo.

Por otro lado, el desarrollo de la confianza es importante para mantener la cooperación entre empresas de la red que son simultáneamente competidoras. Las empresas deben prestar atención a la intensidad de la interacción; es decir, al número de socios dentro de la red con la que se relacionan. Algunos investigadores plantean que los efectos de la confianza y el control sobre los comportamientos de los socios son mutuamente sustituibles y que ese control estricto conducirá inevitablemente a la aparición de desconfianza e, incluso, comportamientos oportunistas. Por el contrario, si los participantes confían completamente entre sí, las medidas de control contractuales desaparecerán.

También existe alguna evidencia de que la confianza y el control son complementarios cuando confiar asegura el cumplimiento de las responsabilidades de los participantes; y de que las medidas de control mejoran la confianza mutua, haciendo más comprensible las acciones de los socios (Inkpen \& Currall, 1998; Zhong et al., 2017; Wang et al., 2019). Las empresas asociadas de diferentes orígenes culturales, arraigadas en diferentes sistemas de gestión y valores, son más propensas a conflictos y malentendidos debido a percepciones erróneas, malas interpretaciones y evaluaciones equivocadas del comportamiento del socio que pueden dañar gravemente la confianza interorganizacional (Hofstede, 1983; Krishnan et al., 2006; Zaheer \& Zaheer, 2006; Ng et al., 2007; Nielsen, 2007; Kwon, 2008). Los miembros de una colaboración interorganizacional están vinculados y así se fortalecen los vínculos. Esto, a su vez, aumenta la previsibilidad del comportamiento entre los socios y fomenta la reciprocidad y la cooperación, que pueden conducir a un mayor grado de confianza (Williamson, 1985; Parkhe, 1993; Zaheer \& Venkatraman, 1995; Inkpen \& Currall, 1998; Ybarra \& Turk, 2009). Hasta la fecha, la investigación ha sido particularmente inconclusa con respecto a la relación entre confianza y control, y existe un debate en curso sobre si los mecanismos de control sustituyen o complementan la 
confianza interorganizacional. La perspectiva de la sustituibilidad se basa en el principio fundamental de la economía de los costos de transacción; es decir, las empresas deben desarrollar mecanismos de control adecuados para inhibir el oportunismo y limitar los costos de transacción (Parkhe, 1993; Gulati, 1995; Nooteboom et al., 1997; Das \& Teng, 1998; Poppo \& Zenger, 2002; Inkpen \& Currall, 1998; Lui \& Ngo, 2004; Cao \& Lumineau, 2015).

En lo expresado, encontramos un escenario en el que la confianza alcanzada entre los socios de una alianza estratégica estaría vinculada con la disminución del control con consecuencias en el desempeño de la alianza. En este contexto, planteamos la siguiente hipótesis:

H2: la confianza entre los socios de las alianzas estratégicas provoca el relajamiento del control en dicha alianza.

\subsubsection{El efecto de la desconfianza en las alianzas estratégicas}

Se define "desconfianza» como una expectativa de acción perjudicial resultante de que uno de los socios actúe a través de capacidades deficientes, motivos negativos y comportamiento dañino sin tener en cuenta los mejores intereses del otro. Algunos autores plantean que la desconfianza se ve como lo opuesto a la confianza, y tanto la confianza como la desconfianza se entienden en términos de comportamiento, prestando poca atención a las intenciones y motivos que las promueven (Lewicki et al., 1998). Sin embargo, existe la propuesta de concebir confianza y desconfianza como constructos separados, pero relacionados, de forma tal que la falta de confianza no significa desconfianza (Rotter, 1971; Hosmer, 1995; Hardin, 2004; McKnight \& Choudhury, 2006).

Dos constructos que se utilizan a menudo en los estudios de desconfianza son la malevolencia, o sea, las preocupaciones sobre el compromiso del socio con el bienestar del asociado; y el descrédito, es decir, las preocupaciones sobre la honestidad y confiabilidad del socio. También se incluye el concepto de incompetencia, lo que sugiere que el asociado teme que el socio no tenga la capacidad de hacer lo que se esperaba (Dimoka, 2010).

Por otro lado, la desconfianza tiene un mayor potencial para predecir un alto nivel de percepción de riesgo e implica la expectativa de resultados negativos. Los riesgos percibidos se refieren a la probabilidad de pérdida o de consecuencias negativas porque la desconfianza puede hacer que las personas se enfrenten a un estado más defensivo, lo que conlleva relaciones de alto riesgo. Se considera a la alta desconfianza como una situación en la que las relaciones de las partes se caracterizan por tener un alto nivel de riesgos percibidos porque hay cosas que podrían salir mal; por tanto, la desconfianza puede verse como antecedente de los riesgos percibidos (McKnight et al., 2017).

Para conceptualizar la confianza y la desconfianza han surgido dos enfoques principales, aunque contradictorios. El primer enfoque, la visión unidimensional asume que la confianza y la desconfianza están en los extremos opuestos de un continuo. En tales modelos, la confianza y la desconfianza se consideran sustitutos perfectos y se excluyen mutuamente. Desde esta perspectiva, aumentar la confianza es todo lo que se necesita para evitar la posibilidad de desconfianza. En contraste, el segundo enfoque, la visión bidimensional, postula que la confianza y la desconfianza son constructos conceptualmente 
distintos que pueden variar independientemente entre sí (Deutsch, 1958; Worchel, 1979; Rotter, 1980; Lewicki \& Bunker, 1996; Lewicki et al., 1998; Luhmann, 2018; 2020). La creciente evidencia empírica apoya la idea de que una alta confianza no es lo mismo que una baja desconfianza. De acuerdo con el enfoque bidimensional, la confianza y la desconfianza son constructos separados; y los elementos que contribuyen al desarrollo de la confianza no son necesariamente los mismos que apoyan el desarrollo de la desconfianza (Cho, 2006; Dimoka, 2010; Connelly et al., 2012; Lumineau, 2017).

En paralelo, algunos investigadores han considerado que la desconfianza no es necesariamente disfuncional e, incluso, puede ser saludable en determinadas circunstancias, reconociendo la importancia de la desconfianza en las relaciones interorganizacionales. A menudo, se necesitan inyecciones sustanciales de desconfianza porque mantiene a la gente alerta y capaz de innovar, evitando así caer en las formas habituales de dependencia entre unos y otros (Luhmann, 2018; 2020). Entre los resultados potencialmente positivos de la desconfianza se observa cómo esta puede funcionar para reducir la complejidad y la vulnerabilidad al considerar probable una conducta indeseable. Por lo tanto, al respaldar la cautela, la vigilancia y la anticipación de una conducta lesiva, la desconfianza puede dar lugar a cuestionamientos constructivos y ayudar a las partes a tomar medidas de protección, pudiendo producir así resultados beneficiosos y consecuencias funcionales. La desconfianza puede ser útil para monitorear el comportamiento, asegurando el cumplimiento y previniendo la explotación. Además, es una forma útil de recopilar información constantemente, cuestionar las interpretaciones individuales y desarrollar una vigilancia saludable (Kee \& Knox, 1970; Lewicki et al., 1998; Kramer, 1999; Atkinson \& Butcher, 2003; Marchand \& Vonk, 2005).

De acuerdo con lo expresado, se aprecia cómo la desconfianza, como constructo particular y diferenciado de la confianza, logra incorporar elementos que cimentan los mecanismos de control exigidos por los socios, lo que nos lleva a plantear la siguiente hipótesis:

H3: la desconfianza entre los socios de las alianzas estratégicas provoca el fortalecimiento del control en las alianzas estratégicas.

\subsection{Marco teórico}

Entre las perspectivas teóricas que fundamentan las investigaciones desarrolladas que tratan sobre el control en las alianzas estratégicas, hemos seleccionado la teoría del costo de transacción (TCE, por sus siglas en inglés) como la base teórica de nuestra investigación por la dependencia de la coincidencia entre las características de las transacciones y estructuras de gobierno para el éxito de la alianza.

\subsubsection{Teoría de costos de transacción (TCE)}

La TCE reemplaza la simple búsqueda de interés propio al presentar oportunismo. Por lo tanto, aunque la mayoría de la gente hará lo que dice y algunos harán más la mayor parte del tiempo, los valores atípicos en los que hay mucho en juego son aquellos sobre los que se establece el comportamiento estratégico. En ese sentido, tanto las consideraciones de 
racionalidad como la búsqueda de intereses personales son importantes a este respecto. El oportunismo, por su parte, se refiere a la búsqueda de intereses personales con engaño. Esto incluye - pero no se limita a - otras formas, como mentir, robar y hacer trampa. En este contexto, la TCE plantea dos supuestos importantes sobre los actores económicos: 1) racionalidad limitada y 2) oportunismo; así como tres dimensiones clave de las transacciones económicas: 1) especificidad de activos, 2) frecuencia y 3) incertidumbre (Williamson, 1985; Rindfleisch, 2019).

Los estudiosos de los costos de transacción entienden la confianza como un modo de gobernanza que reduce los costos de transacción al limitar el oportunismo; mientras que los teóricos del intercambio social conceptualizan la confianza como un factor contextual que hace que la coordinación en las alianzas sea más eficiente a medida que se disminuye el miedo al oportunismo, se enriquece la comunicación y se amplía el alcance de la relación. Si bien estas perspectivas teóricas ofrecen diferentes explicaciones, coinciden en la posición de que la confianza conduce al desempeño de la alianza (Young-Ybarra \& Wiersema, 1999; Muthusamy et al., 2007; Ybarra \& Turk 2009). Williamson $(1985,2010)$ propone tres atributos principales mediante los cuales se puede describir cada modo de gobernanza: 1) la intensidad de los incentivos (que se mide por el grado en que una etapa tecnológicamente separable de la actividad económica se apropia de sus ganancias netas); 2) la autoridad administrativa y el control (que tiene una relación con la autonomía de una etapa tanto en los aspectos operativos como de inversión, así como en los controles de procedimiento — rutinas, procedimientos contables—-) y 3) régimen de derecho contractual, donde el enfoque está puesto en los dos primeros atributos. Así, en este último caso, de acuerdo con el incentivo de dos valores — fuerte o débil—, se describe el modo de mercado (propiedad independiente) como uno que tiene una fuerte intensidad de incentivos dentro de cada etapa y controles administrativos débiles en la interfaz contractual; mientras que la jerarquía (propiedad unificada) tiene incentivos débiles dentro de cada etapa y un fuerte control administrativo en la interfaz.

Además, la TCE considera que el principal problema de la organización económica es el de la adaptación, y distingue entre la adaptación autónoma y coordinada. La cuestión de qué transacciones se organizan y según qué modo de gobernanza depende de cómo los atributos de las diferentes transacciones plantean diferentes necesidades de adaptación, por un lado; y cómo los modos alternativos de gobernanza están, en razón de sus diferentes atributos, bien o mal calificados para responder a distintas necesidades de adaptación.

\section{Discusión y resultados}

Al profundizar en los aspectos relacionados a la influencia de la confianza y la desconfianza en la formulación de mecanismos de control orientados al éxito de las alianzas estratégicas, nuestra revisión de la literatura ha identificado la ausencia de estudios orientados a desmitificar el control como garantía de éxito de las alianzas estratégicas, y también el rol de la confianza y la desconfianza como constructos independientes empleados por las firmas en su propósito de fortalecer su ilusión de control sobre la alianza.

La literatura nos muestra el deseo de las firmas de controlar procesos, procedimientos y resultados, llevando a los socios de las alianzas a implementar 
mecanismos de control con la expectativa de que la relación funcione de acuerdo con los contratos suscritos. Al mismo tiempo, en la definición de estos contratos, los atributos de confianza y desconfianza influencian en la incorporación de sus cláusulas. Sin embargo, la literatura revisada no explica por qué la gran mayoría de estos acuerdos no logran ejecutarse en los tiempos señalados en el contrato; ni por qué se incumplen las condiciones pactadas, a pesar de los mecanismos de control incorporados por las partes (Vaydia, 2011; Bedford et al., 2016; Lumineau, 2017).

En este contexto, la TCE no solo tiene muchas aplicaciones dentro del campo de la organización industrial, sino también dentro de la mayoría de los campos aplicados de la economía, que incluyen los de trabajo, finanzas públicas, sistemas económicos comparativos, y desarrollo y reforma económica. La TCE tiene un alcance amplio porque cualquier problema que surja o pueda reformularse como un problema de contratación puede ser examinado para obtener ventajas, en términos de economía de costos de transacción, para una discusión de las aplicaciones con referencias a la literatura relevante (Macher \& Richman, 2008). La confianza y la desconfianza como elementos moderadores del control influyen en el desempeño de las alianzas estratégicas. Para ello, se propone un modelo teórico fundamentado en la TCE, el cual plantea la elección de la estructura de gobierno adecuada para limitar la amenaza del comportamiento oportunista del socio y postula el análisis de la actividad económica a través del contrato, centrándose en la transacción y definiendo límites basados en los términos contractuales (Williamson, 2012). Estas transacciones se encuentran definidas en las cláusulas de los contratos, que responden a percepciones de confianza, desconfianza o ambas.

La literatura nos muestra que las firmas tienden a ser más confiadas cuando sienten que tienen un adecuado nivel de control, considerándolo como un proceso regulatorio por el cual los elementos de un sistema son más predecibles a través de la formalización de estándares en la búsqueda del objetivo deseado. No obstante, la relación de estos mecanismos con el desempeño de las alianzas estratégicas se encuentra aún en discusión.

Finalmente, con base en lo expresado, se propone el siguiente modelo conceptual (figura 1), que asume la influencia de la confianza y la desconfianza como variables independientes moderadoras en la construcción de mecanismos de control generados para gestionar el desempeño de las alianzas estratégicas. En este modelo, que busca explicar la ambigüedad de la relación entre los mecanismos de control —particularmente, la confianza y la desconfianza- y el desempeño de las alianzas estratégicas, el desempeño de la alianza estratégica es la variable dependiente.

Figura 1. Modelo teórico conceptual

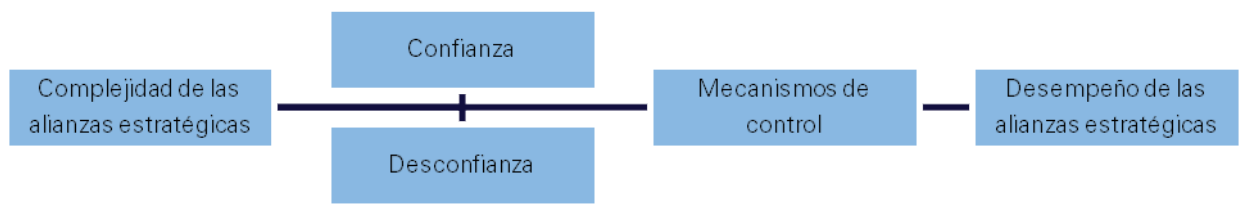

Fuente: elaboración propia. 


\section{CONCLUSIONES}

La revisión de la literatura muestra cómo los investigadores han buscado establecer la relación entre los mecanismos de control y el éxito de las alianzas estratégicas basados en teorías orientadas a 1) identificar las relaciones interorganizacionales (teoría institucional), 2) la atribución de responsabilidades a los actores intervinientes y la necesidad de reporte (teoría de agencia), y 3) la búsqueda de la eficiencia con la adecuada gestión de costos (TCE). Sin embargo, se identifica la ausencia de investigaciones que expliquen el alcance del control en el desempeño de las alianzas estratégicas, considerando el portafolio de consistencias que se presentan en las relaciones interorganizacionales basadas en la confianza y la desconfianza. Asimismo, a pesar de la coincidencia de los autores en mostrar la confianza como atributo esencial en la formación de las alianzas estratégicas, recientemente se ha incluido a la desconfianza como un atributo independiente que modera la construcción del control.

Todo arreglo de colaboración enfrenta la amenaza potencial del oportunismo, lo que hace que las empresas participantes sean vulnerables a las acciones de sus socios. Por esto, la confianza entre los socios de la alianza, como variable clave en la relación del desempeño del control, se basa en el razonamiento del costo de la acción. De esa manera, la confianza puede considerarse un medio para asegurar la cooperación e inhibir el comportamiento oportunista.

A su vez, la desconfianza puede ser saludable en determinadas circunstancias porque mantiene a la firma organización alerta y le da la capacidad de innovar para no caer en las formas habituales de codependencia entre las empresas. Al respaldar la cautela, vigilancia y anticipación de una conducta lesiva, la desconfianza puede dar lugar a cuestionamientos constructivos y ayudar a las partes a tomar medidas de protección, pudiendo producir resultados beneficiosos con consecuencias funcionales.

La utilidad de los mecanismos de control como base para mitigar el oportunismo puede ser patente, pero relativamente efímera, en el turbulento contexto competitivo e incierto de la mayoría de las alianzas estratégicas. Nuestro análisis encuentra la correlación cualitativa en el uso de controles de gestión incluidos en los contratos basados en la TCE, que se utilizan para estudiar las prácticas de control de gestión y gobernanza empleadas para mitigar el riesgo que implican las alianzas entre empresas.

Asimismo, se ha concluido que la noción de éxito es complicada y varía con el tiempo; además, las percepciones de lo que significa tener éxito en una alianza pueden diferir entre los socios específicos que participan en ella. En general, el éxito puede definirse como la minimización de fricciones y la reducción de los costos de transacción; por ejemplo, los costos del contrato se reducen cuando las empresas confían entre sí. Por esta razón, consideramos relevante la aplicación de estudios empíricos que confirmen la relación moderadora de la confianza y la desconfianza como variables independientes en el desempeño de las alianzas estratégicas. 


\section{bibliografía}

Albers, S., Wohlgezogen, F., \& Zajac, E. J.

2016 Strategic alliance structures: An organization design perspective. Journal of Management, 42(3), 582-614.

\section{Anderson, L. A., \& Dedrick, R. F.}

1990 Development of the Trust in

Physician scale: a measure to assess

interpersonal trust in patient-physician relationships. Psychological Reports, 67(3, suplemento), 1091-1100

\section{Anand, B. N., \& Khanna,T.}

2000 Do firms learn to create value? The case of alliances. Strategic Management Journal, 21(3), 295-315.

\section{Atkinson, S., \& Butcher, D.}

2003, Trust in the context of management

11-13 relationships: an empirical study. Society de abril for Advancement of Management (SAM) International Business Conference. Orlando, Florida.

\section{Ballou, R. H.}

2004 Logística: Administración de la cadena de suministro. Pearson Educación.

Baltes, M. M., \& Baltes, P. B. (eds.).

1986 The psychology of control and aging.

L. Erlbaum Associates.

\section{Bandura, A.}

1977 Self-efficacy: toward a unifying theory of behavioral change. Psychological Review, 84(2), 191-215.

\section{Bhattacharya, R., Devinney,}

T. M., \& Pillutla, M. M.

\section{Bills, K. L., Hayne, C., \& Stein, S. E.}

Collaborating with Competitors:

Mechanisms that Mitigate Coopetitive

Tensions Among Member Firms in

Accounting Associations and Networks [borrador]

\section{Bills, K. L., Hayne, C., Stein,}

\section{S. E., \& Hatfield, R. C.}

Collaborating with competitors: How do small firm accounting associations and networks successfully manage coopetitive tensions? Contemporary Accounting Research, 38(1), 545-584.

\section{Bleeke, J., \& Ernst, D.}

1991 The way to win in cross-border alliances. Harvard Business Review, 69(6)، 127-135

Brim, J. A.

Social network correlates of avowed happiness. Journal of Nervous and Mental Disease, 158(6), 432-439.

\section{Cacioppo, J.T., \& Berntson, G. G.}

Relationship between attitudes and evaluative space: A critical review, with emphasis on the separability of positive and negative substrates. Psychological Bulletin, 115(3), 401.

\section{Cao, M., \& Zhang, 0.}

collaborative advantage and firm 


\section{bibliografía}

performance. Journal of Operations

Management, 29(3), 163-180.

Cao, Z., \& Lumineau, F.

2015

Revisiting the interplay between

contractual and relational governance:

A qualitative and meta-analytic

investigation. Journal of Operations

Management, 33-34(1), 15-42.

Cardinal, L. B., Sitkin, S. B., \&

Long, C. P.

2004

Balancing and rebalancing in the creation

and evolution of organizational control.

Organization Science, 15(4), 411-431.

\section{Castelfranchi, C., \& Falcone, R.}

Trust and control: A dialectic link. Applied

Artificial Intelligence, 14(8), 799-823.

Ceptureanu, E. G., Ceptureanu, S., Rădulescu, V., \& lonescu, S.

What Makes Coopetition Successful?

An Inter-Organizational Side Analysis on

Coopetition Critical Success Factors in Oil and Gas Distribution Networks. Energies,

11(12), 3447.

\section{Chen, H., \& Chen, T. J.}

Governance structures in strategic

alliances: transaction cost versus resourcebased perspective. Journal of World Business, 38(1), 1-14.

Chen, G., Zhang, G., \& Xie, Y. M. transaction costs in project alliances:

Disaggregated analysis. Journal of

Management in Engineering, 31(4), 15-42.

https://doi.org/10.1061/(ASCE)ME.1943-

5479.0000259
1998

\section{Child, J., \& Faulkner, D.}

Strategies of Cooperation: Managing

Alliances, Networks, and Joint Ventures.

Oxford University.

Cho, J.

2006

The mechanism of trust and distrust

formation and their relational

outcomes. Journal of Retailing, 82(1),

25-35

\section{Christoffersen, J.}

2013

A review of antecedents of international strategic alliance performance: synthesized evidence and new directions for core constructs. International Journal of Management Reviews, 15(1), 66-85.

\section{Connelly, B. L., Miller, T., \& Devers, C. E.}

Under a cloud of suspicion: Trust, distrust, and their interactive effect in interorganizational contracting. Strategic Management Journal, 33(7), 820-833.

Dacin, M. T., Hitt, M. A., \& Levitas, $\mathrm{E}$.

Selecting partners for successful international alliances: Examination of US and Korean firms. Journal of World Business, 32(1), 3-16.

\section{Dahlquist, S. H.}

Interfirm marketing alliance: understanding the influences of complementarity, compatibility, and combinative capacity on success. International Journal of Strategic Business Alliances, 4(2-3), 167-183.

\section{Das, T. K., \& Teng, B. S.}

Between Trust and Control Developing

Confidence in Partner Cooperation in 


\section{bibliografía}

Alliances. The Academy of Management

Review, 23(3), 491-512.

\section{Das, T. K., \& Teng, B. S.}

2001 Trust, control and risk in strategic alliances: An integrated framework. Organization Studies, 22(2), 251-283.

\section{De Grande, P.}

2014 Reflexiones sobre la interpretación

del concepto de locus de control en investigación social. Pensando Psicología, 10(17), 127-134.

\section{Dekker, H. C.}

2004 Control of inter-organizational relationships: evidence on appropriation concerns and coordination requirements. Accounting, Organizations and Society, 29(1), 27-49.

\section{Dekker, H. C., \& Van den} Abbeele, A.

2010 Organizational Learning and Interfirm Control: The Effects of Partner Search and Prior Exchange Experiences. Organization Science, 21(6), 1233-1250.

\section{Deutsch, M.}

1958 Trust and suspicion. Journal of Conflict Resolution, 2(4), 265-279.

\section{Dimoka, A.}

2010 What does the brain tell us about trust and distrust? Evidence from a functional neuroimaging study. MIS Quarterly, 34(2), 373-396.

Ding, Q., Akoorie, M. E., \& Pavlovich, K.

2009 A critical review of three theoretical approaches on knowledge transfer in cooperative alliances. International Journal of Business and Management, 4(1), 47-55.
Doney, P. M., \& Cannon, J. P.

1997 An examination of the nature of trust in buyer-seller relationships. Journal of Marketing, 61(2), 35-51.

\section{Dyer, J. H., \& Chu, W.}

2000 The determinants of trust in supplierautomaker relationships in the US, Japan and Korea. Journal of International Business Studies, 31(2), 259-285.

\section{Dyer, J. H., \& Singh, H.}

1998 The relational view: Cooperative strategy and sources of interorganizational competitive advantage. Academy of Management Review, 23(4), 360-379.

\section{Dyer, J. H., Singh, H., \&} Hesterly, W. S.

2018 The relational view revisited: A dynamic perspective on value creation and value capture. Strategic Management Journal, 39(12), 3140-3162.

Duysters, G., Kok, G., \& Vaandrager, $\mathbf{M}$.

1999 Crafting successful strategic technology partnerships. R\&D Management, 29(4), 343-351.

Fryxell, G. E., Dooley, R. S., \& Vryza, M.

2002 After the ink dries: the interaction of trust and control in US-based international joint ventures. Journal of Management Studies, 39(6), 865-886.

Gomes, E., Barnes, B. R., \& Mahmood, T.

2015 A 22 year review of strategic alliance research in the leading management journals. International Business Review, 25(1), 15-27. 


\section{bibliografía}

Gomes, E., Weber, Y., Brown, C., \& Tarba, S.Y.

2011

Mergers, Acquisitions and Strategic

Alliances: Understanding the Process.

Macmillan International Higher Education.

Grewal, R., Chakravarty, A., \&

\section{Saini, A.}

2010

Governance mechanisms in business-to-

business electronic markets. Journal of

Marketing, 74(4), 45-62.

\section{Gulati, R.}

Does familiarity breed trust? The implications of repeated ties for contractual choice in alliances. Academy of Management Journal, 38(1), 85-112.

\section{Gulati, R.}

Alliances and networks. Strategic

Management Journal, 19(4), 293-317.

Gulati, R., Nohria, N., \& Zaheer, A.

Strategic networks. Strategic Management Journal, 21(3), 203-215.

\section{Gulati, R., \& Nickerson, J. A.}

Interorganizational trust, governance

choice, and exchange performance.

Organization Science, 19(5), 688-708.

\section{Gulati, R., Sytch, M., \&}

\section{Mehrotra, P.}

Breaking up is never easy: Planning for exit in a strategic alliance. California Management Review, 50(4), 147-163.

\section{Guo, S.-L., Lumineau, F., \& Lewicki, R. J.}

2017 Revisiting the foundations of organizational distrust. Foundations and Trends.

Microeconomics, 1(1), 1-88.
Hameed, W., \& Abbott, C.

2017

Critical review of the success factors of strategic alliances in the UK construction industry. En 13th International

Postgraduate Research Conference 2017:

Conference Proceedings (pp. 15-26).

University of Salford.

Hardin, R. (ed.).

2004

Distrust. Russell Sage Foundation.

\section{Harris, A. Z.}

2006

Interorganizational trust. En O. Shenkar and J. Reuer (eds.), Handbook of Strategic Alliances (pp. 169-197). Thousand Oak, California: Sage.

\section{Heckhausen, J., \& Schulz, R.}

A life-span theory of control. Psychological Review, 102(2), 284-304.

Hofstede, G.

1983

The cultural relativity of organizational practices and theories. Journal of International Business Studies, 14(2), 75-89.

Hitt, M. A., Dacin, M. T., Tyler, B. B., \& Park, D.

Understanding the differences in Korean and us Executives' strategic orientations. Strategic Management Journal, 18(2), 159-167.

Hitt, M. A., Dacin, M. T., Levitas, E., Arregle, J. L., \& Borza, A.

Partner selection in emerging and developed market contexts: Resourcebased and organizational learning perspectives. Academy of Management Journal, 43(3), 449-467. 


\section{bibliografía}

Hosmer, L.T.

1995

Trust: The connecting link between

organizational theory and philosophical

ethics. Academy of Management

Review, 20(2), 379-403.

\section{Inkpen, A. C., \& Beamish, P. W.}

1997 Knowledge, bargaining power, and

the instability of international joint

ventures. Academy of Management

Review, 22(1), 177-202.

Inkpen, A. C., \& Currall, S. C.

The nature, antecedents, and

consequences of joint venture trust.

Journal of International Management, 4(1),

$1-20$.

Jiang, X., Li, M., Gao, S., Bao,

Y., \& Jiang, $F$.

2013

Managing knowledge leakage in strategic

alliances: The effects of trust and

formal contracts. Industrial Marketing

Management, 42(6), 983-991.

\section{Kachelmeier, S. J., \& Van}

Landuyt, B. W.

Prompting the benefit of the doubt: The joint effect of auditor-client social bonds and measurement uncertainty on audit adjustments. Journal of Accounting Research, 55(4), 963-994.

Kale, P., Singh, H., \& Perlmutter, H.

Learning and protection of proprietary assets in strategic alliances: Building relational capital. Strategic Management Journal, 21(3), 217-237.

Kalkman, J. P., \& de Waard, E. J.

projects: Finding the middle way between

trust and control. International Journal of

Project Management, 35(5), 889-899.

Kapmeier, F., \& Struben, J.

2017

Understanding the Dynamics of Alliance

Capabilities. Hochschule Reutlingen.

Kee, H. W., \& Knox, R. E.

1970

Conceptual and methodological

considerations in the study of trust

and suspicion. Journal of conflict

resolution, 14(3), 357-366.

\section{Khanna, T.}

1998

The scope of alliances. Organization

Science, 9(3), 340-355.

\section{Killing, P.}

2013 Strategies for Joint Venture Success (RLE international business). Routledge, Taylor \& Francis Group.

\section{Kramer, R. M.}

1999 Trust and distrust in organizations: Emerging perspectives, enduring questions. Annual Review of Psychology, 50(1), 569-598

Kramer, R. M., \& Lewicki, R. J.

2010 Repairing and enhancing trust:

Approaches to reducing organizational trust deficits. Academy of Management Annals, 4(1), 245-277.

Krishnan, R., Martin, X., \& Noorderhaven, N. G.

2006 When does trust matter to alliance performance? Academy of Management Journal, 49(5), 894-917. 


\section{bibliografía}

Lewicki, R. J., McAllister, D. J., \&

Bies, R. J.

2004

Trust and distrust: New relationships

and realities. Academy of Management

Review, 23(3), 438-458

\section{Li, L., Qian, G., \& Qian, Z.}

2013

Do partners in international strategic

alliances share resources, costs, and

risks? Journal of Business Research, 66(4), 489-498.

\section{Liu, Y., Luo, Y., \& Liu, T.}

Governing buyer-supplier relationships

through transactional and relational

mechanisms: Evidence from China. Journal

of Operations Management, 27(4), 294-309.

Liu, W., Sidhu, A. K., Beacom, A. M., \& Valente, T.

Social Network Theory. John Wiley \& Sons.

Luhmann, $\mathbf{N}$.

Trust and Power. John Wiley \& Sons.

\section{Lui, S. S., \& Ngo, H.Y.}

The role of trust and contractual

safeguards on cooperation in non-equity alliances. Journal of Management, 30(4), 471-485.

\section{Lumineau, F.}

How contracts influence trust and distrust. Journal of Management, 43(5), 1553-1577.

\section{Lumineau, F., \& Henderson, J. E.} contractual governance on the negotiation strategy in buyer-supplier disputes. Journal of Operations Management, 30(5), 382-395.
2008 Lunnan, R., \& Haugland, S. A.

Predicting and measuring alliance performance: A multidimensional analysis. Strategic Management Journal, 29(5), 545-556.

Ma, C., Yang, Z., Yao, Z., Fisher, G., \& Fang, E. E.

The effect of strategic alliance resource accumulation and process characteristics on new product success: Exploration of international high-tech strategic alliances in China. Industrial Marketing Management, 41(3), 469-480.

Macher, J. T., \& Richman, B. D.

Transaction cost economics: An assessment of empirical research in the social sciences. Business and Politics, 10(1), 1-63.

\section{Mahama, $\mathrm{H}$.}

Management control systems, cooperation and performance in strategic supply relationships: A survey in the mines. Management Accounting Research, 17(3), 315-339.

\section{Marchand, M. A., \& Vonk, R.}

The process of becoming suspicious of ulterior motives. Social Cognition, 23(3), 242-256.

Mayer, R. C., Davis, J. H., \& Schoorman, F. D.

An integrative model of organizational trust. Academy of Management Review, 20(3), 709-734

\section{McKnight, D. H., \& Choudhury, V.}




\section{bibliografía}

International Conference on Electronic

Commerce (pp. 482-491). Nueva York:

Association of Computing Machinery.

McKnight, D. H., Lankton, N. K., Nicolaou, A., \& Price, J.

2017

Distinguishing the effects of B2B

information quality, system quality,

and service outcome quality on trust

and distrust. The Journal of Strategic

Information Systems, 26(2), 118-141.

Meier, M., Lütkewitte, M., Mellewigt, T., \& Decker, C.

How managers can build trust in strategic

alliances: a meta-analysis on the central

trust-building mechanisms. Journal of

Business Economics, 86(3), 229-257.

\section{Mohr, J., \& Spekman, R.}

Characteristics of partnership success:

Partnership attributes, communication behavior and conflict resolution techniques, Strategic

Management Journal, 15(2), 135-152.

\section{Muthusamy, S. K., \& White, M. A.}

2005 Learning and knowledge transfer in

strategic alliances: a social exchange

view. Organization Studies, 26(3), 415-441.

\section{Muthusamy, S., White, M., \&} Carr, A.

An Empirical Examination of the

Role of Social Exchanges in Alliance

Performance. Journal of Managerial

Issues, 19(1), 53-75.

\section{Nielsen, B. B., \& Nielsen, S.}

Nooteboom, B., Berger, H., \&

Noorderhaven, N. G.

1997

Effects of trust and governance on

relational risk. Academy of Management

Journal, 40(2), 308-338.

\section{Park, S. H., \& Ungson, G. R.}

200

Interfirm rivalry and managerial complexity:

A conceptual framework of alliance

failure. Organization Science, 12(1), 37-53.

Parkhe, A.

1993

Strategic alliance structuring: A game theoretic and transaction cost examination of interfirm cooperation. Academy of Management Journal, 36(4), 794-829.

\section{Parkhe, A.}

1998 Building trust in international alliances. Journal of World Business, 33(4), 417-437.

\section{Poppo, L., \& Zenger, T.}

Do formal contracts and relational governance function as substitutes or complements? Strategic Management Journal, 23(8), 707-725.

\section{Rindfleisch, A.}

2019 Transaction cost theory: past, present and future. Academy of Marketing Science Review, 10(5), 85-97.

Ring, P. S., \& Van de Ven, A. H. Developmental processes of cooperative interorganizational relationships. Academy of Management Review, 19(1), 90-118.

Rotter, J. B.

trust. American Psychologist, 26(5), 443-452. 


\section{bibliografía}

Ruíz-Vargas, J. M.

1933

Atención y control: modelos y problemas para una integración teórica. Revista de psicología general y aplicada: Revista de la Federación Española de Asociaciones de Psicología, 46(2), 125-137.

\section{Schaan, J. L. F.}

Parent control and joint venture success:

The case of Mexico [tesis doctoral, University of Western Ontario]. Digitalized Theses, 1252. https://ir.lib.uwo.ca/ digitizedtheses/1252

Schulz, R., Heckhausen, J., \& Locher, J. L.

Adult development, control, and adaptive functioning. Journal of Social Issues, 47(4), 177-196.

\section{Shyam Kumar, M. V.}

Differential Gains Between Partners

in Joint Ventures: Role of Resource

Appropriation and Private Benefits.

Organization Science, 21(1), 232-248.

Sitkin, S. B., \& Roth, N. L.

Explaining the limited effectiveness of legalistic "remedies" for trust/distrust. Organization Science, 4(3), 367-392.

Stoner, J. A. F., Freeman, R. E., \& Gilbert, D. R.

Administración. Pearson Educación.

Tadelis, S., \& Williamson, O. E.
Transaction cost economics. En R. Gibbons

y J. Roberts (eds.), The Handbook of

Organizational Economics (pp. 159-190).

Princeton, Nueva Jersey: Princeton

University Press.
2013

Taleb, N. N.

Antifrágil: las cosas que se benefician del desorden (trad. G. S. Barberán, pp. 178-179) Paidós.

Teece, D. J.

Competition, Cooperation, and Innovation: Organizational Arrangements for Regimes of Rapid Technological Progress. Journal of Economic Behavior and Organization, 18(1), 1-25.

\section{Tomkins, $\mathrm{C}$.}

Interdependencies, trust and information in relationships, alliances and networks. Accounting, Organizations and Society, 26(2), 161-191.

Tse, Y. K., Zhang, M., Tan, K. H., Pawar, K., \& Fernandes, K.

Managing quality risk in supply chain to drive firm's performance: The roles of control mechanisms. Journal of Business Research, 97, 291-303.

\section{Vaidya, S.}

Understanding Strategic Alliances: An Integrated Framework. Journal of Management Policy and Practice, 12(6), 90-100.

\section{Walgenbach, P.}

The production of distrust by means of producing trust. Organization Studies, 22(4), 693-714.

\section{Wang, D., Fu, H., \& Fang, S.}

The efficacy of trust for the governance of uncertainty and opportunism in megaprojects: The moderating role of contractual control. Engineering, 


\section{bibliografía}

Williamson, O. E.

1979

Transaction-cost economics: the

governance of contractual relations.

The Journal of Law and Economics, 22(2), 233-261.

\section{Williamson, O. E.}

1985 Assessing contract. Journal of Law,

Economics, \& Organization, 1(1), 177-208.

Williamson, O. E.

1993 Calculativeness, trust, and economic organization. The Journal of Law and Economics, 36(1, parte 2), 453-486.

Williamson, O. E.

Transaction cost economics and business administration. Scandinavian Journal of Management, 21(1), 19-40.

\section{Williamson, O. E.}

2010 Transaction cost economics: The natural progression. American Economic Review, 100(3), 673-690.

\section{Williamson, O. E.}

2012 Transaction Cost Economics: What

Are the Questions [borrador]. https://

businessinnovation.berkeley.edu/wpcontent/uploads/businessinnovationarchive/WilliamsonSeminar/

williamson040512.pdf

\section{Williamson, O. E.}

2014 The transaction cost economics project. Montenegrin Journal of Economics, 10(1), 7-12.

Ybarra, C. E., \& Turk, T. A.

The evolution of trust in information technology alliances. The Journal of High Technology Management Research, 20(1), 62-74.
Young-Ybarra, C., \& Wiersema, M.

1999 Strategic flexibility in information technology alliances: The influence of transaction cost economics and social exchange theory. Organization Science, 10(4), 439-459.

\section{Zaheer, A., McEvily, B., \& Perrone, $\mathrm{V}$.}

1998 Does trust matter? Exploring the effects of interorganizational and interpersonal trust on performance. Organization Science, 9(2), 141-159

\section{Zaheer, A., \& Venkatraman, N.}

Relational governance as an

interorganizational strategy: An empirical

test of the role of trust in economic exchange. Strategic Management Journal, 16(5), 373-392.

\section{Zaheer, S., \& Zaheer, A.}

Trust across borders. Journal of International Business Studies, 37(1),

21-29.

Zhang, Q., \& Zhou, Z. K.

2013 Governing interfirm knowledge transfer in the Chinese market: The interplay of formal and informal mechanisms. Industrial Marketing Management, 42(5), 783-791.

Zhong, W., Su, C., Peng, J., \& Yang, $\mathbf{Z}$.

2017 Trust in interorganizational relationships: A meta-analytic integration. Journal of Management, 43(4), 1050-1075.

Zollo, M., Reuer, J. J., \& Singh, H.

Interorganizational routines and performance in strategic alliances. Organization Science, 13(6), 701-713. 


\section{bibliografía}

Zucker, L. G.

1986 Production of trust: Institutional sources of economic structure, 1840-1920. Research in Organizational Behavior, 8, 54-111.

Fecha de recepción: 16 de septiembre de 2021 Fecha de aceptación: 26 de noviembre de 2021

Fecha de publicación: 4 de diciembre de 2021 